\title{
Meet Our Section Editor
}

\author{
Prof. Fabio Stella \\ Department of Informatics, Systems and Communication \\ University of Milan-Bicocca, Milan \\ Italy
}

Fabio Stella is an Associate Professor in the Department of Informatics, Systems and Communication at the University of Milano-Bicocca. He received his Ph.D. in Computational Mathematics and Operations Research at the University of Milano in 1995. He worked as an assistant professor in the University of Milano from 1993 to 1998 before joining the University of Milano-Bicocca in 1998. He worked as a research assistant in the EEC IMPROD project devoted to improve the quality of semiconductors using the Data Mining methodology. From 1998 to 2000 he worked as a research leader in the Pirelli P-Vision project to improve tyre's performance using Data Mining. From 2000 to 2002 he was a consultant for the Risk Management of Banca Intesa. His main research activities include; Bayesian networks, continuous-time Bayesian networks, Feedforward neural networks, on-line portfolio selection algorithms and probabilistic topic models. He serves as a referee for a number of Interna-

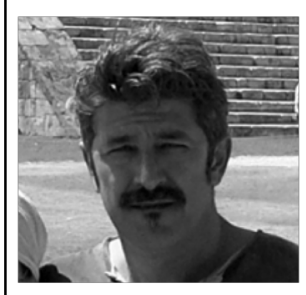

F. Stella tional Journals; Journal of Approximate Reasoning, Expert Systems with Applications, BioData Mining, Journal of Biomedical Informatics, Pattern Recognition Letters, Data Mining and Knowledge Discovery, Information Processing and Management, European Journal of Operations Research and Quantitative Finance. From 2015, he is a member of the Programme Committee of the Uncertainty in Artificial Intelligence conference. In 2017, he becomes the member of the Programme Committee of the RecSys Conference, International Conference on Machine Learning, Neural Information Processing Conference. From 2006, Fabio Stella teaches Probability and Statistics for Bachelor of Science in Informatics and from 2007 he teaches Data and Text Mining for Master of Science in Informatics at the University of Milan-Bicocca. He also teaches Data and Text Mining in the foreign Universities. Furthermore, starting from 2018 he teaches Machine Learning for the Master of Science in Data Science. In 2016, he developed the Massive Online Open Course pathway entitled: "Introduction to Data Mining". The pathway is freely available on the EduOpen platform and consists of three courses.

\section{SELECTED PUBLICATIONS}

[1] Liu, M.; Stella, F.; Hommersom, A.; Lucas, P.J.F.; Boer, L.; Bischoff, E. A comparison between discrete and continuous time Bayesian networks in learning from clinical time series data with irregularity. Artif. Intell. Med., 2019, 95, 104-117. doi: 10.1016/j.artmed.2018.10.002.

[2] Villa, S.; Stella, F. Learning continuous time Bayesian networks in non-stationary domains. J. Artif. Intell. Res., 2016, $57(1)$, 1-37.

[3] Acerbi, E.; Viganò, E.; Poidinger, M.; Mortellaro, A.; Zelante, T.; Stella, F. Continuous time Bayesian networks identify Prdm1 as a negative regulator of TH17 cell differentiation in humans. Sci. Rep., 2016, 6, 23128.

[4] Codecasa, D.; Stella, F. Learning continuous time Bayesian network classifiers. Int J Approx. Reason., 2014, 55(8), 1728-1746. Available from: http://dx.doi.org/10.1016/j.ijar.2014.05.005.

[5] Acerbi, E.; Zelante, T.; Narang V.; Stella, F. Gene network inference using continuous time Bayesian networks: a comparative study and application to Th17 cell differentiation. BMC Bioinformatics, 2014, 15(387), doi: 10.1186/s12859-014-0387-x

[6] Gatti, E.; Luciani, D.; Stella, F. A continuous time Bayesian network model for cardiogenic heart failure. Flex. Serv. Manuf. J., 2012, 24(4), doi: 10.1007/s10696-011-9131-2.

[7] Tuana, G.; Volpato, V.; Ricciardi-Castagnoli, P.; Zolezzi, F.; Stella, F.; Foti, M. Classification of dendritic cell phenotypes from gene expression data. BMC Immunol., 2011, 12, 50. doi: 10.1186/1471-2172-12-50.

[8] Fraccalvieri, D.; Pandini, A.; Stella, F.; Bonati, L. Conformational and functional analysis of molecular dynamics trajectories by Self-Organising Maps. BMC Bioinformatics, 2011, 12, 158. 